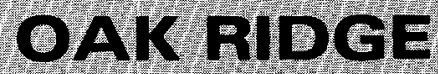

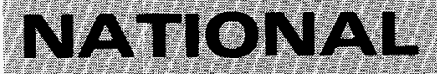

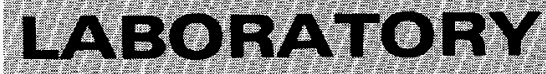

MARTIN MARUETLA

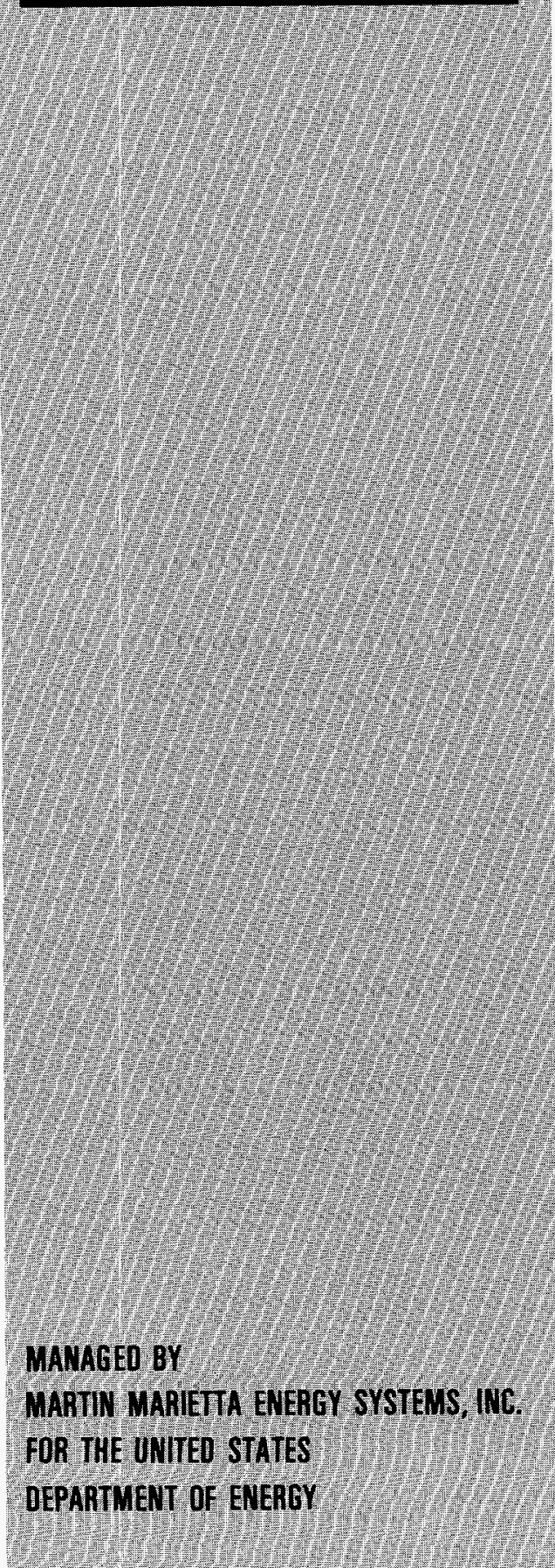

\section{Beta Testing the Intel Paragon MP}

Thomas H. Dunigan 
This report has been reproduced directly from the best available copy.

Available to DOE and DOE contractors from the Office of Scientific and Technical Information, P.O. Box 62, Oak Ridge, TN 37831; prices available from (615) 576-8401, FTS 626-8401.

Available to the public from the National Technical Information Service, U.S. Department of Commerce, 5285 Port Royal Ad., Springtield, VA 22161.

This report was prepared as an account of work sponsored by an agency of the United States Government. Neither the United States Government nor any agency thereof, nor any of their employees, makes any warranty, express or implied, or assumes any legal liability or responsibility for the accuracy, completeness, or usefulness of any information, apparatus, product, or process disclosed, or represents that its use would not infringe privately owned rights. Reference herein to any specific commercial product, process, or service by trade name, trademark, manufacturer, or otherwise, does not necessarily constitute or imply its endorsement, recommendation, or favoring by the United States Government or any agency thereof. The views and opinions of authors expressed herein do not necessarily state or reflect those of the United States Government or any agency thereof. 


\section{DISCLAIMER}

Portions of this document may be illegible in electronic image products. Images are produced from the best available original document. 
Computer Science and Mathematics Division

Mathematical Sciences Section

BETA TESTING THE INTEL PARAGON MP

Thomas H. Dunigan

\author{
Mathematical Sciences Section \\ Oak Ridge National Laboratory \\ P.O. Box 2008, Bldg. 6012 \\ Oak Ridge, TN 37831-6367 \\ thd@ornl.gov
}

Date Published: June 1995

Research was supported by the Office of Scientific Computing of the Office of Energy Research, U.S. Department of Energy.

\author{
Prepared by the \\ Oak Ridge National Laboratory \\ Oak Ridge, Tennessee 37831 \\ managed by \\ Martin Marietta Energy Systems, Inc. \\ for the \\ U.S. DEPARTMENT OF ENERGY \\ under Contract No. DE-AC05-84OR21400
}




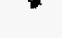




\section{Contents}

1 Introduction ..................... 1

2 Paragon MP Architecture . . . . . . . . . . . . . 1

3 Performance ...................... 3

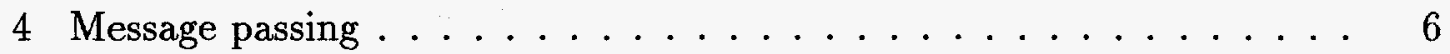

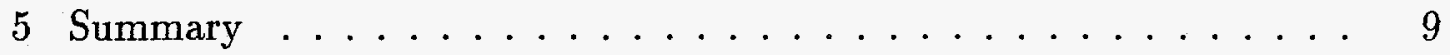

6 References . . . . . . . . . . . . . . . . . 9

A Timeline .......................... . . 11

B Comparative Architectures. . . . . . . . . . . . . 11 


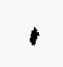

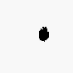




\title{
BETA TESTING THE INTEL PARAGON MP
}

\author{
Thomas H. Dunigan
}

\begin{abstract}
This report summarizes the third phase of a Cooperative Research and Development Agreement between Oak Ridge National Laboratory and Intel in evaluating a 28-node Intel Paragon MP system. An MP node consists of three $50-\mathrm{MHz}$ i860XP's sharing a common bus to memory and to the mesh communications interface. The performance of the shared-memory MP node is measured and compared with other shared-memory multiprocessors. Bus contention is measured between processors and with message passing. Recent improvements in message passing and $\mathrm{I} / \mathrm{O}$ are also reported.
\end{abstract}




\section{Introduction}

The Department of Energy selected Oak Ridge National Laboratory (ORNL) as one of its high performance computing centers as part of the government's High Performance Computing and Communications (HPCC) initiative. The initiative provided ORNL with funds to procure a massively parallel computer and to support various Grand Challenge applications. ORNL selected Intel to provide the massively parallel computer for the HPCC project. Intel has developed a family of distributed-memory multiprocessors, starting with the iPSC/1 hypercube in 1986. The Intel multiprocessors are members of a growing market of parallel processing systems that are being used by researchers and commercial organizations to tackle increasingly complex computational tasks. A Cooperative Research and Development Agreement (CRADA) between ORNL and Intel specified the staging of increasingly more powerful versions of its new Paragon multiprocessor. As part of the agreement, ORNL would receive pre-production models of the Paragon and assist in beta testing and product development. This report summarizes the results of the third and final phase of the CRADA, the test and evaluation of the Paragon MP.

In 1994, the first Paragon MP was delivered to ORNL. The Paragon MP extended the computational power of the Paragon by providing three i860XP processors with a shared memory on each node of the communication mesh. Appendix A provides a time-line of the events during the test and evaluation of the Paragon MP system. This report details the performance of the shared-memory node board and evaluates the performance of several parallel applications on the Paragon MP. Computational, shared-memory, and communication performance were measured with synthetic benčhmarks, application kernels, and a few parallel applications. The Paragon's shared-memory performance is compared with the performance of other shared-memory parallel processors.

In the following section, the Paragon MP architecture is summarized. Section 3 describes the performance of the shared-memory MP node and compares its performance to other shared-memory architectures. In section 4 , recent improvements in message passing and $\mathrm{I} / \mathrm{O}$ are reported.

\section{Paragon MP Architecture}

The Intel Paragon system is a mesh-connected parallel processor. In the first member of the Paragon family, the GP system, each node on the mesh consists of two $50 \mathrm{MHz}$ i860XP processors, memory, and communication hardware. One processor is used for computation, and the second processor is for communication. 
A Paragon MP node consists of three $50 \mathrm{MHz}$ i860XP processors, memory, and communication hardware (Figure 2.1). The nodes are interconnected by a 2-D mesh with $175 \mathrm{MB} /$ second communication channels and a per-hop latency of only 40 ns. The nodes are logically subdivided into service nodes, compute nodes, and I/O nodes (Figure 2.1). The service nodes appear as a single host and support time-sharing through the OSF operating system. The compute nodes run OSF or SUNMOS. The I/O nodes are connected to local networks and arrays of disks (RAID) and provide a UNIX file system, swap/paging space, and a Parallel File System (PFS).

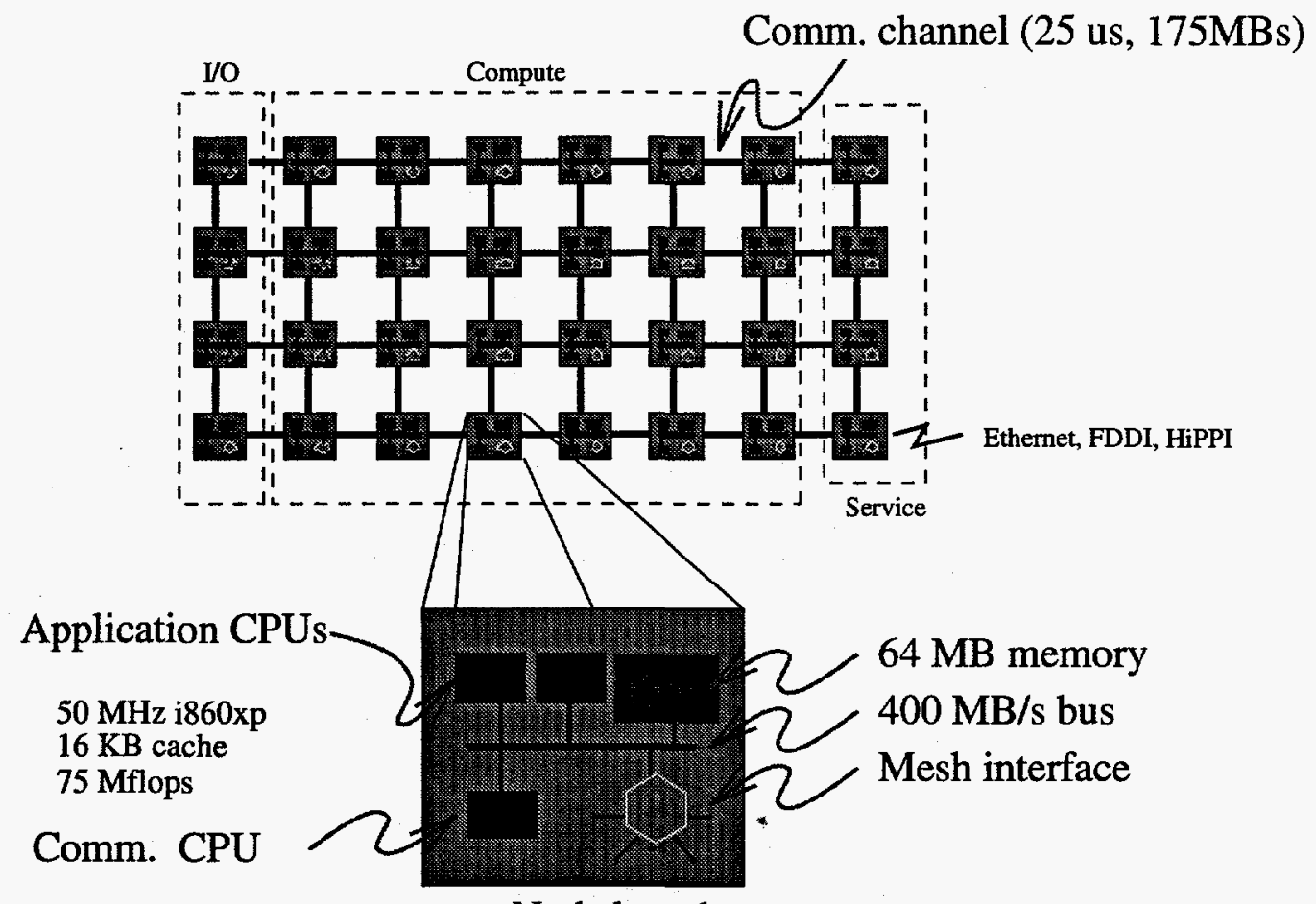

Node board

Figure 2.1: $M P$ node board.

Each i860XP has its own $16 \mathrm{~KB}$ data and instruction cache, and each node has at least $64 \mathrm{MB}$ of memory. The bus interconnecting the processors, meshinterface, and memory operates at $400 \mathrm{MB} /$ second. The $50 \mathrm{MHz}$ i860XP is a super-scalar architecture capable of a peak $75 \mathrm{Mflops}$ (double precision). Typical FORTRAN performance is only 11 Mflops ([2]). Early designs of the MP proposed five CPUs with L2 cache, but cost-performance analyses dictated the three-CPU configuration and no secondary cache. Intel's analyses showed the bus bandwidth to the local memory would not support five CPUs efficiently. Also the threeCPU configuration provided more board real-estate for memory than the five- 
CPU design and the five-CPU design would have required using only every other backplane slot.

Message-passing libraries (NX, PVM, MPI, SUNMOS) are provided for internode communication. A node is the smallest addressable unit in the messagepassing architecture. (Conceptually, each processor might be addressable in the message-passing software, but Intel's early design analyses favored node addressing.) Typically, one processor on each node is designated as the communication processor, leaving two processors for computational work. The compilers can provide automatic parallelization of the processors on a node, or a threaded library and compiler directives are provided for explicit parallelization.

\section{Performance}

In this section, we look at the performance of the shared-memory node. We measure CPU memory bandwidth and the effects of bus contention when multiple CPUs and message passing compete for the limited bus bandwidth. We compare the shared-memory performance and multi-threading primitives to other shared-memory multiprocessors, finally comparing performance of some parallel benchmark kernels.

An MP node has three CPUs, memory, and mesh-controller sharing a 400 $\mathrm{MB} /$ second bus (Figure 2.1). A $50 \mathrm{MHz}$ i860XP is specified as being able to generate $400 \mathrm{MB} /$ second of memory traffic. Clearly, the MP node architecture is likely to be bus limited. Large caches on each processor could mitigate the limited bandwidth, but the data cache is not large (16KB). With $90 \%$ to $95 \%$ cache hit rates and typical cache write-back rates, one can expect that only $15 \%$ to $25 \%$ of a CPU's memory requests actually generate a memory operation. In principle, the $400 \mathrm{MB} /$ second bus could support four or five i860XPs. Programs can be contrived to demonstrate either extreme: where all data requests are satisfied from cache, and linear speed-up is possible; or where little or no cache hits occur, and the node runs at the speed of one (or less) processor.

To measure actual memory bandwidth performance, we used a small unrolled assembler loop that did quad load's ( $p f d q$ ) from memory. The memory locations were "pre-touched" to eliminate any virtual memory effects. A single CPU sustained a memory access rate of $251 \mathrm{MB} /$ second, considerably less than the $400 \mathrm{MB} /$ second specification. If we ran our test concurrently on two CPUs, the aggregate rate was still only $237 \mathrm{MB} /$ second. If all three CPUs on the node concurrently accessed memory, the aggregate rate was $246 \mathrm{MB} /$ second (Table 3.1). Table 3.1 also shows the data rates for a $\mathrm{C}$ double-precision inner-product 
on one, two, and three CPUs. The vector lengths are too long to be contained in the $16 \mathrm{~KB}$ cache, and speedups are sublinear due to bus contention. (The $\mathrm{C}$ inner-product for cacheable vectors gave linear speedups with a data rate of 88 $\mathrm{MB} /$ second per CPU.)

\begin{tabular}{|l||r|r|r|r|}
\hline \multicolumn{6}{|c|}{ MP memory bandwidth (MBs) } \\
\hline CPUs & 1 & 2 & 3 & Sum \\
\hline \hline pfldq-1 & 251 & & & 251 \\
\hline pfldq-2 & 118 & 119 & & 237 \\
\hline pfldq-3 & 80 & 82 & 84 & 246 \\
\hline ddot-1 & 39 & & & 39 \\
\hline ddot-2 & 25 & 25 & & 50 \\
\hline ddot-3 & 21 & 21 & 21 & 63 \\
\hline
\end{tabular}

Table 3.1: Memory bandwidth consumption.

We compared the MP shared-memory node board with other shared-memory multiprocessors. We compared thread and fork creation, lock and unlock, barriers, and concurrent update of shared variable (no locks). The i860XP has no hardware "atomic" operations, so locks are implemented by software. Table $3.2 \mathrm{com}$ pares single processor performance of the $50 \mathrm{MHz}$ i860XP with single processors on the KSR, BBN, and Sequent. The KSR is ring-based shared-memory multiprocessor using a $20 \mathrm{MHz}$ custom processor. The Sequent Symmetry is bus-based shared-memory multiprocessor using $16 \mathrm{MHz} 386$ processors. The BBN TC2000 is a cascaded-switch based shared-memory multiprocessor using $20 \mathrm{MHz} \mathrm{M} 88000$ processors (see Appendix B). The performance of the i860XP and the Paragon

\begin{tabular}{|l||r|r|r|r|}
\hline \multicolumn{5}{|c|}{ Time on one CPU $(\mu \mathbf{s})$} \\
\hline & MP & KSR & BBN & Sequent \\
\hline \hline fork/wait & 50,000 & 108,000 & 44,000 & 14,000 \\
\hline thread/join & 1,191 & 130 & 79 & 26 \\
\hline lock/unlock & 13 & 3 & 8 & 10 \\
\hline barrier & 13 & 39 & 11 & 10 \\
\hline hotspot & 0.12 & 0.41 & 1.3 & 1.4 \\
\hline
\end{tabular}

Table 3.2: Single processor performance of shared memory.

thread library is comparable to the other multiprocessors. The thread/join times are slower, but the Intel programming model is such that threads are usually only created once at the start of the application.

Table 3.3 compares the performance of three processors for an MP node board with three processors for the KSR, BBN, and Sequent. The MP node 
compares reasonably to the three-processor performance of the other multiprocessors. There appear to be no architecture or implementation penalties in the MP thread primitives for one, two, or three processors.

\begin{tabular}{|l||r|r|r|r|}
\hline \multicolumn{5}{|c|}{ Time on three CPUs $(\mu$ s $)$} \\
\hline & MP & KSR & BBN & Sequent \\
\hline \hline lock/unlock & 104 & 17 & 20 & 38 \\
\hline barrier & 110 & 119 & 38 & 24 \\
\hline hotspot & 0.81 & 2.5 & 5.3 & 6.4 \\
\hline
\end{tabular}

Table 3.3: Three processor performance.

Table 3.4 compares the MP node performance over a set of application kernels in $\mathrm{C}$ and FORTRAN. The same copy of the code was run on each multiprocessor, and the codes have not been tuned. The numeric integration kernels effectively operate from cache, so near linear speed-up is achieved. The Cholesky code is a little more memory intensive, and the slower MP performance results from lock and bus contention. The HiTC kernel is based on a double-precision complex ZAXPY. Using Intel's ZAXPY from the kmath library, the serial code runs at 36 Mflops. The vectors in the ZAXPY exceed the i860XP cache size, and bus contention prevents the parallel HiTC kernel from achieving any speedup on an MP node. Another version of the HiTC kernel, modeling only one atom per cell, has small enough vectors that near linear speedups can be attained.

\begin{tabular}{|l||r|r|r|}
\hline \multicolumn{4}{|c|}{ Speedup on three CPUs } \\
\hline & MP & KSR & Sequent \\
\hline \hline Integration (C) & 2.9 & 2.9 & 3.0 \\
\hline Jacobi iteration (C) & 2.7 & 2.8 & 3.0 \\
\hline Cholesky (1K $\times 1 \mathrm{k})(\mathrm{C})$ & 2.1 & 3.0 & 2.9 \\
\hline Integration (F) & 2.9 & 2.9 & 3.0 \\
\hline HiTC kernel (F) & 1.0 & 2.9 & 2.9 \\
\hline
\end{tabular}

Table 3.4: Speedup on three CPUs for various application kernels.

To this point, we have considered only a single node board. In parallel applications, each Paragon node will communicate with other nodes in the mesh in solving a parallel application. The expected configuration is to use one CPU on each node board as a communication processor. To see the effect of communication and computation competing for the bus, we added a communication thread to our $p f l d q$ test. In the absence of computational activity, the communication thread ran at $119 \mathrm{MB} /$ second, using an echo test to an adjacent node. In the absence of communication, the $p f l d q$ ran at $252 \mathrm{MB} /$ second. With one $p f l d q$ thread 
and one communication thread running concurrently for identical durations, the aggregate data rate was $177 \mathrm{MB} /$ second. The communication thread garnered 31 $\mathrm{MB} /$ second, and the $p f l d q$ thread achieved about $146 \mathrm{MB} /$ second. So the limited bus speed can slow both computation and communication.

Table 3.5 summarizes speedups of the FORTRAN NAS parallel benchmarks on a Pargon MP (using two compute processors and one communication processor) as reported by Intel in the Spring of 1995 (OSF R1.3). Speedups are relative to a Paragon GP (one compute processor and one communication processor). The class B versions represent larger problems (larger arrays or more iterations). The NAS results are consistent with the early results of ORNL "grand challenge" applications on the Paragon MP. The material science parallel application realized a speed-up of 1.7 on the MP versus the GP. However, the shallow-water kernel showed little speedup on the MP, but that kernel is characterized by low data re-use.

\begin{tabular}{|l||l|}
\hline Program & Speedup \\
\hline \hline EP class A & $1.74-1.91$ \\
\hline EP class B & $1.94-2.00$ \\
\hline FT class A & $1.20-1.42$ \\
\hline FT class B & $1.24-1.42$ \\
\hline MG class A & $1.21-1.37$ \\
\hline MG class B & $1.32-1.39$ \\
\hline
\end{tabular}

Table 3.5: Intel reported speedups of Pargon MP verus GP for FORTRAN NAS Parallel Benchmarks.

\section{Message passing}

Our beta testing concentrated primarily on the shared-memory features of the $\mathrm{MP}$, but we also re-evaluated message-passing performance and I/O. Most of our production research is conducted using Intel's OSF on the compute nodes, but we also continue to evaluate SUNMOS. Our communication tests uncovered several performance anomalies. Data rates were poor if message sizes were not a multiple of 32 bytes, and data rates of one-to- $n$ communication degraded as $n$ increased. Intel corrected the anomalies in subsequent software releases. Message-passing performance (latency and bandwidth) improved with each release of software. For nearest neighbor communication, we are currently measuring latencies of 25 to $30 \mu \mathrm{s}$ for zero-length messages, and data rates of nearly $171 \mathrm{MB} /$ second for one MB messages. These numbers were measured under OSF 1.0.4 R1_3 and 
SUNMOS 1.6.2 and are much faster than those we reported just last year ([4]). Per-hop delay is nearly negligible. The additional delay for going corner to corner on the 1024-node MP Paragon $(16 \times 64)$ is less than $3 \mu \mathrm{s}$.

The compute nodes can be configured in a "turbo" mode, where all three processors are used as computation processors. Communication tasks are handled with context switches. Our early tests showed that communication performance was several orders of magnitude slower in turbo mode. However, recent software releases have greatly improved turbo mode communication. Latency slows to $75 \mu \mathrm{s}$, and bandwidth is reduced to $109 \mathrm{MB} /$ second. Figure 4.1 compares the message-passing performance (transfer time) for OSF, SUNMOS, and OSF in turbo mode. Transfer times are half the round-trip time for 1,000 repetitions of an echo test to a neighboring node.

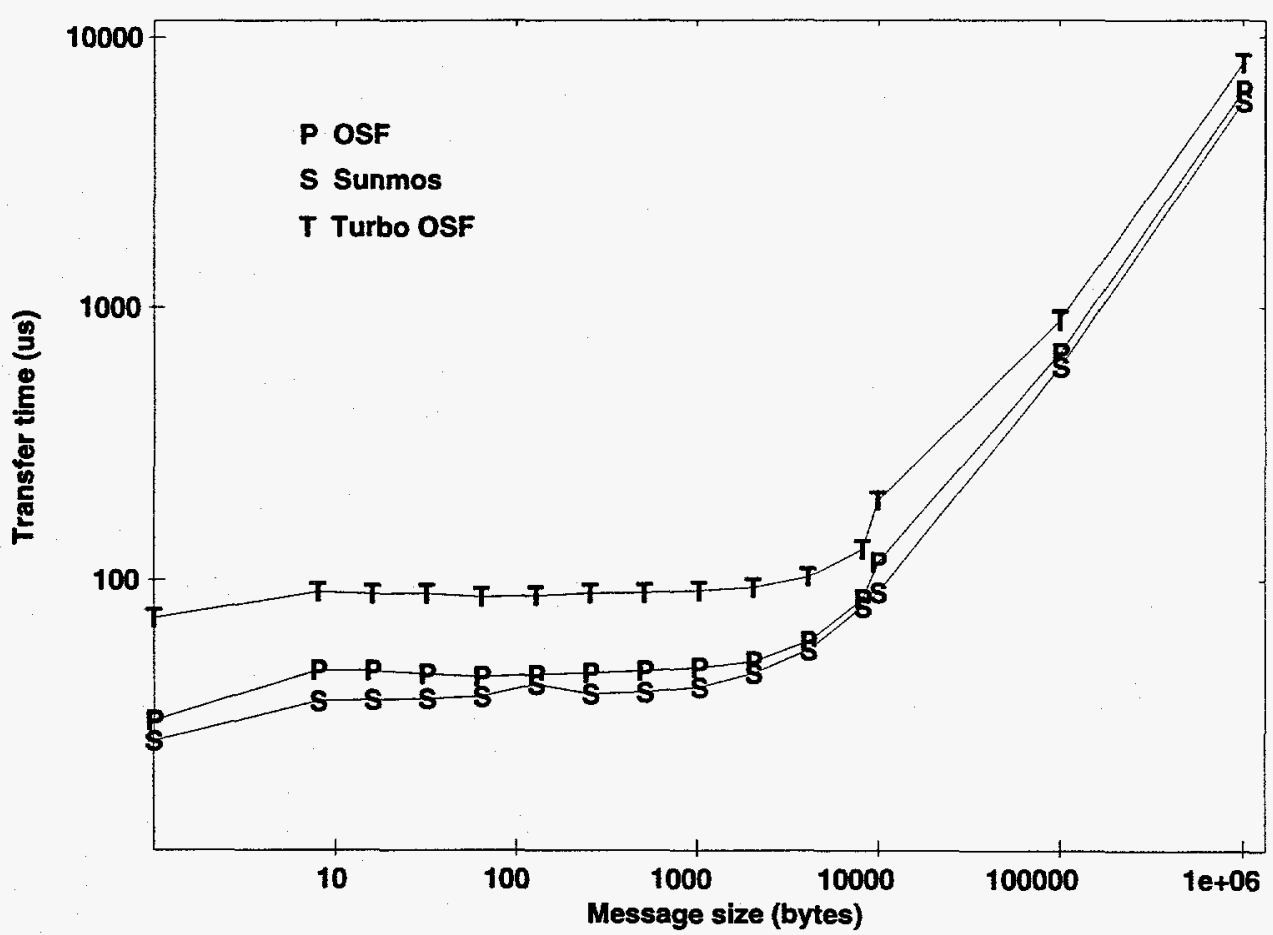

Figure 4.1: Message transfer time for the Paragon MP.

Our CRADA analysis also re-evaluated I/O performance. The Paragon OSF provides both a standard UNIX file system and a larger, high performance parallel file system (PFS). PFS is typically configured across a set of $I / O$ nodes and disks. The PFS is striped across one or more I/O nodes and their disk RAID arrays and appears to the UNIX system as a separate mountable file system (e.g., /pfs). The striping factor is $64 \mathrm{~KB}$. PFS performance improves with additional $\mathrm{I} / \mathrm{O}$ nodes and larger block sizes and is limited on each node by the throughput of the OSF 
NORMA IPC communication. IPC is used by OSF to communicate between the compute nodes and $1 / O$ nodes. The IPC communication is in turn transported by the Paragon message passing hardware and software. Using $2 \mathrm{MB}$ records to $64 \mathrm{I} / \mathrm{O}$ nodes, a single MP node achieves an $18 \mathrm{MB} /$ second read rate and a 36 $\mathrm{MB} /$ second write rate (Figure 4.2). Figure 4.2 also shows that the NORMA IPC data rate between adjacent $\mathrm{MP}$ nodes peaks at about $45 \mathrm{MB} / \mathrm{second}$, and the read and write I/O data rates follow the same basic curve as the IPC performance. The IPC performance probably limits I/O performance, since the IPC data rate is well below the $171 \mathrm{MB} /$ second data rate available from the underlying mesh.

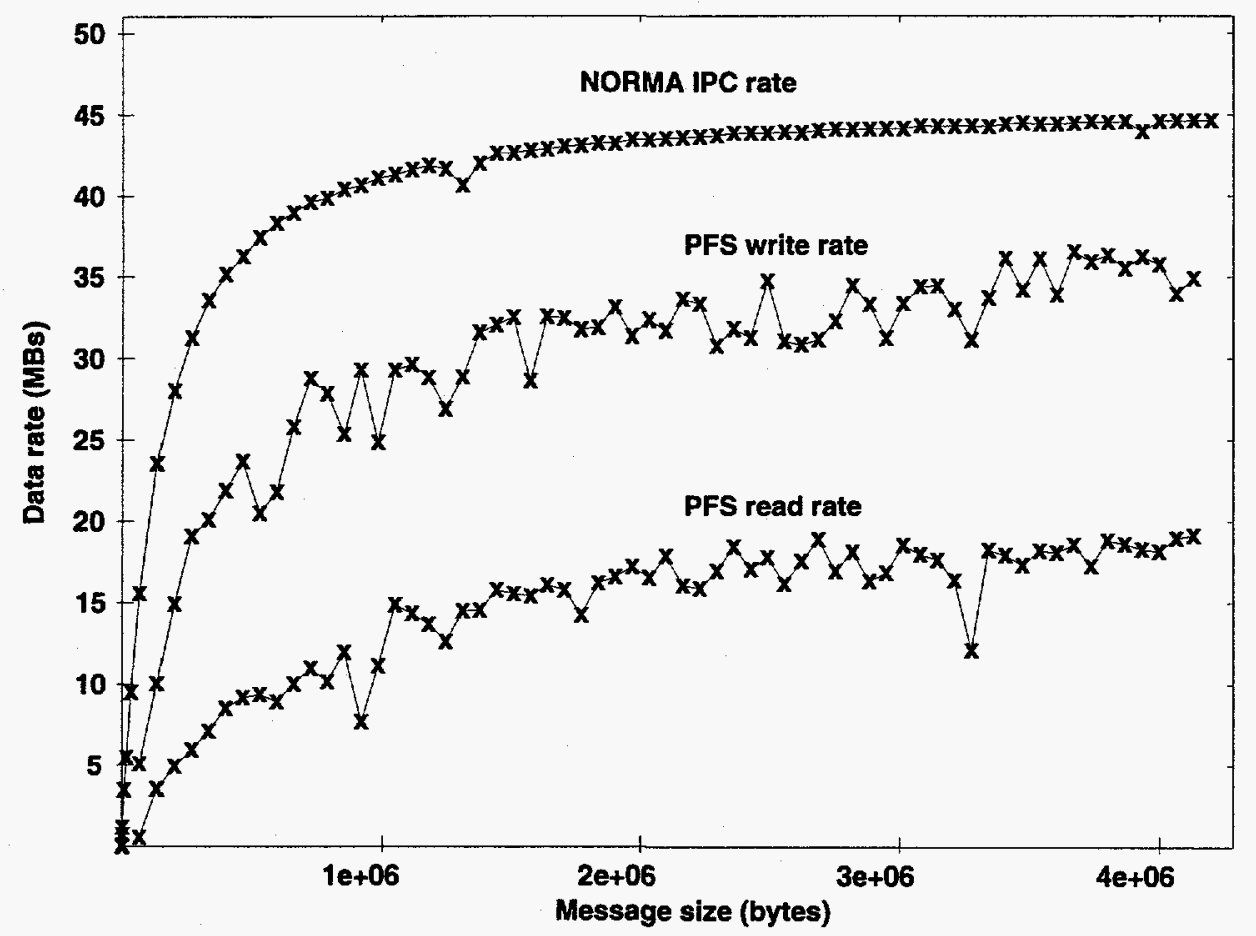

Figure 4.2: Paragon MP NORMA IPC rate and PFS read/write data rates.

Figure 4.3 shows aggregate read data rate using 16, 32, and $64 \mathrm{I} / \mathrm{O}$ nodes, with from 1 to 128 compute nodes doing concurrent I/O. Aggregate read data rates of $95 \mathrm{MB} /$ second are achieved with $64 \mathrm{I} / \mathrm{O}$ nodes and 128 compute nodes, an improvement over earlier results ([4]). The PFS tests use $64 \mathrm{~KB}$ blocks, and each compute processor reads a $32 \mathrm{MB}$ section of a file. Our PFS tests use the M_RECORD mode of gopen(), and open and close times are included in the timings. 


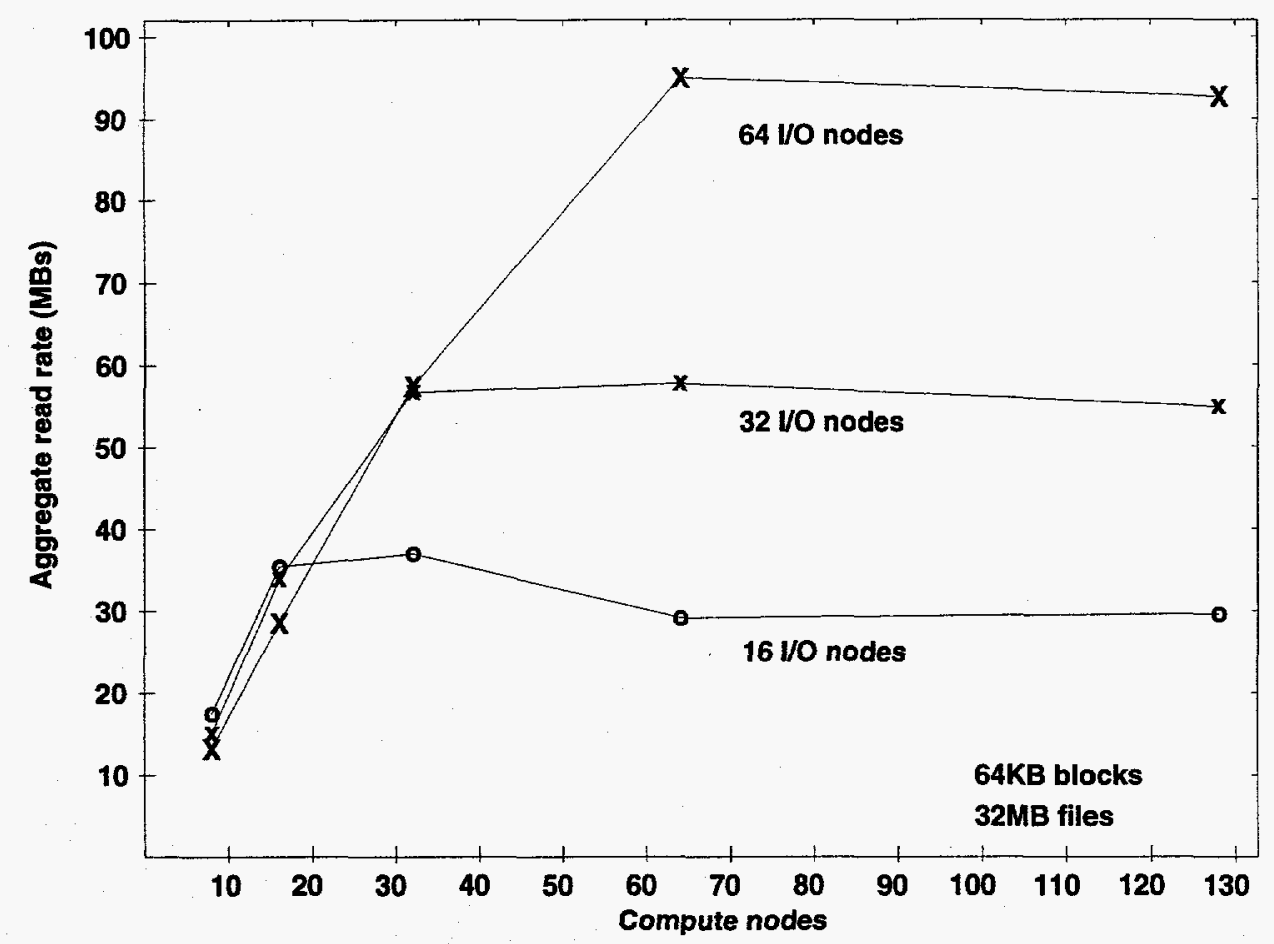

Figure 4.3: Aggregate read data rate for varying compute and $I / O$ nodes.

\section{Summary}

The final phase of the ORNL/Intel CRADA provided value to both parties, Intel getting feedback from early users and performance analyses, and ORNL getting an opportunity to do leading-edge computer science and computational science. The shared-memory performance of the Paragon MP is competitive with other shared-memory multiprocessors. The limited bus bandwidth of each MP node requires that the application programmer exploit data locality to garner noticeable speedups. The automatic parallelization compilers help in utilizing the multiple processor nodes, but in complex programs, the application programmer usually needs to assist in the parallelization process. Message-passing performance and I/O continue to improve. The 96-node MP Paragon CRADA machine continues to be a valuable computational resource for ORNL, even after delivery of the production 1024-node MP Paragon in January, 1995.

\section{References}

[1] J. Dongarra. Performance of various computers using standard linear equations software. Technical report, University of Tennessee, January 1991. CS- 
89-85.

[2] J. Dongarra. Performance of various computers using standard linear equations software. Technical report, University of Tennessee, January 1993. CS89-85.

[3] T. H. Dunigan. Kendall Square multiprocessor: Early experiences and peformance. Technical report, Oak Ridge National Laboratory, 1992. ORNL/TM12065.

[4] T. H. Dunigan. Early experiences and performance of the intel paragon. Technical report, Oak Ridge National Laboratory, 1993. ORNL/TM-12194.

[5] R. P. LaRowe and C. S. Ellis. Experimental comparison of memory management policies for numa multiprocessors. Technical report, Duke University, April 1990. CS-1990-10.

[6] R. D. Rettberg, W. R. Crowther, P. P. Carvey, and R. S. Tomlinson. The monarch parallel processor hardware design. Computer, 23:18-30, April 1990. 


\section{Appendix}

\section{A. Timeline}

May, 1993. Inititial "planned" delivery of beta MP system to ORNL.

July, 1993. Intel changes from MP5 to MP3.

October, 1993. Fat-node programming model selected.

April, 1994. Training on MP at Beaverton.

May, 1994. 10-node MP delivered to ORNL.

June, 1994. MP training at ORNL and parallel FORTRAN delivered.

July, 1994. MP expanded to 28 nodes and additional training.

October, 1994. Applications testing on 1024-node MP system (XPS150) at Beaverton.

January, 1995. MP expanded to 96 nodes and XPS150 delivered.

\section{B. Comparative Architectures.}

A node on the Paragon MP supports a shared-memory architecture with three i860XP processors sharing a $400 \mathrm{MB} /$ second bus and memory. The report compares shared memory performance of an MP node with several shared-memory multiprocessors. A summary of the shared-memory multiprocessors compared with the MP node follows.

\section{BBN TC2000}

The BBN TC2000 at Argonne National Laboratory (ANL) is a 45 processor shared-memory parallel processor. Each processor is a Motorola 88000 running at $20 \mathrm{MHz}$ with $16 \mathrm{MB}$ of memory fronted by a $16 \mathrm{~KB}$ data cache and a $16 \mathrm{~KB}$ instruction cache. All of the memories are interconnected by a 2-stage 8-way switch. The system can be expanded up to 512 processors. The Uniform programming environment (under $\mathrm{nX} 2.0 .6$ ) provides the program with both local and explicitly allocated shared memory. The shared memory may be allocated in another processor's memory, and thus a non-uniform memory access (NUMA) model is supported. In the absence of contention, a remote reference typically takes less 
than two microseconds, and a single channel of the switch has a bandwidth of $40 \mathrm{MBs}$ [6]. The architecture could be used with other memory management policies [5]. Compiles on the BBN were done with $-\mathrm{O}$-lus. LINPACK $100 \times 100$ double-precision on a single processor was 1.0 Mflops using -OLM -autoinline. Dhrystone (v1.0) was 19.4 Mips.

\section{Kendall Square}

The Kendall Square uses custom-designed $20 \mathrm{MHz}$ processors that share memory on a one gigabyte per second ring. Each processor has a $256 \mathrm{~KB}$ cache, and the global memory is managed as a cache. A single processor generates a maximum of $40 \mathrm{MBs}$ against the ring. LINPACK $100 \times 100$ double-precision on a single processor was 15 Mflops [3].

\section{Sequent Symmetry}

The 26 processor Sequent Symmetry located at ANL is based on 80386/387 processors (16 MHz) with a Weitek 3167 floating point co-processor. Each processor has a $64 \mathrm{~KB}$ cache, and $32 \mathrm{MB}$ of memory is shared by all processors on a $54 \mathrm{MBs}$ bus. The maximum configuration is 30 processors. The processors run Dynix 3.1.2, and compiles were done using -O. LINPACK $100 \times 100$ double-precision on a single processor was 0.37 Mflops [1]. Dhrystone (v1.0) was 3.6 Mips. Processor $4.8 \mathrm{MBs}$ versus a $26 \mathrm{MBs}$ bus. 
ORNL/TM-12830

\section{INTERNAL DISTRIBUTION}

1. T. S. Darland

2. J. J. Dongarra

3-7. T. H. Dunigan

8. G. A. Geist

9. K. L. Kliewer

10. M. R. Leuze

11. C. E. Oliver

12. R. T. Primm

13-17. S. A. Raby
18-22. M. R. Leuze

23-27. R. F. Sincovec

28. P. H. Worley

29. Central Research Library

30. ORNL Patent Office

31. K-25 Appl Tech Library

32. Y-12 Technical Library

33. Laboratory Records - RC

34-35. Laboratory Records Department

\section{EXTERNAL DISTRIBUTION}

36. Cleve Ashcraft, Boeing Computer Services, P.O. Box 24346, M/S 7L-21, Seattle, WA 98124-0346

37. Robert G. Babb, Oregon Graduate Institute, CSE Department, 19600 N.W. von Neumann Drive, Beaverton, OR 97006-1999

38. Lawrence J. Baker, Exxon Production Research Company, P.O. Box 2189, Houston, TX 77252-2189

39. Clive Baillie, Physics Department, Campus Box 390, University of Colorado, Boulder, CO 80309

40. Jesse L. Barlow, Department of Computer Science, 220 Pond Laboratory, Pennsylvania State University, University Park, PA 16802-6106

41. Edward H. Barsis, Computer Science and Mathematics, P. O. Box 5800, Sandia National Laboratories, Albuquerque, NM 87185

42. Professor Larry Dowdy, Computer Science Department, Vanderbilt University, Nashville, TN 37235

43. Chris Bischof, Mathematics and Computer Science Division, Argonne National Laboratory, 9700 South Cass Avenue, Argonne, IL 60439

44. Ake Bjorck, Department of Mathematics, Linkoping University, S-581 83 Linkoping, Sweden

45. James C. Browne, Department of Computer Science, University of Texas, Austin, TX 78712

46. Bill L. Buzbee, Scientific Computing Division, National Center for Atmospheric Research, P.O. Box 3000, Boulder, CO 80307

47. Donald A. Calahan, Department of Electrical and Computer Engineering, University of Michigan, Ann Arbor, MI 48109 
48. Thomas A. Callcot, Director Science Alliance, 53 Turner House, University of Tennessee, Knoxville, TN 37996

49. Ian Cavers, Department of Computer Science, University of British Columbia, Vancouver, British Columbia V6T 1W5, Canada

50. Tony Chan, Department of Mathematics, University of California, Los Angeles, 405 Hilgard Avenue, Los Angeles, CA 90024

51. Jagdish Chandra, Army Research Office, P.O. Box 12211, Research Triangle Park, NC 27709

52. Siddhartha Chatterjee, RIACS, MAIL STOP T045-1, NASA Ames Research Center, Moffett Field, CA 94035-1000

53. Eleanor Chu, Department of Mathematics and Statistics, University of Guelph, Guelph, Ontario, Canada N1G 2W1

54. Melvyn Ciment, National Science Foundation, 1800 G Street N.W., Washington, DC 20550

55. Tom Coleman, Department of Computer Science, Cornell University, Ithaca, NY 14853

56. Paul Concus, Mathematics and Computing, Lawrence Berkeley Laboratory, Berkeley, CA 94720

57. Andy Conn, IBM T. J. Watson Research Center, P.O. Box 218, Yorktown Heights, NY 10598

58. John M. Conroy, Supercomputer Research Center, 17100 Science Drive, Bowie, MD 20715-4300

59. Jane K. Cullum, IBM T. J. Watson Research Center, P.O. Box 218, Yorktown Heights, NY 10598

60. George Cybenko, Center for Supercomputing Research and Development, University of Illinois, 104 S. Wright Street, Urbana, IL 61801-2932

61. George J. Davis, Department of Mathematics, Georgia State University, Atlanta, GA 30303

62. Tim A. Davis, Computer and Information Sciences Department, 301 CSE, University of Florida, Gainesville, FL 32611-2024

63. John J. Dorning, Department of Nuclear Engineering Physics, Thornton Hall, McCormick Road, University of Virginia, Charlottesville, VA 22901

64. Iain Duff, Numerical Analysis Group, Central Computing Department, Atlas Centre, Rutherford Appleton Laboratory, Didcot, Oxon OX11 0QX, England

65. Patricia Eberlein, Department of Computer Science, SUNY at Buffalo, Buffalo, NY 14260

66. Albert M. Erisman, Boeing Computer Services, Engineering Technology Applications, P.O. Box 24346, M/S 7L-20, Seattle, WA 98124-0346

67. Geoffrey C. Fox, Northeast Parallel Architectures Center, 111 College Place, Syracuse University, Syracuse, NY 13244-4100

68. Robert E. Funderlic, Department of Computer Science, North Carolina State University, Raleigh, NC 27650 
69. Professor Dennis B. Gannon, Computer Science Department, Indiana University, Bloomington, IN 47401

70. David M. Gay, Bell Laboratories, 600 Mountain Avenue, Murray Hill, NJ 07974

71. C. William Gear, NEC Research Institute, 4 Independence Way, Princeton, NJ 08540

72. W. Morven Gentleman, Division of Electrical Engineering, National Research Council, Building M-50, Room 344, Montreal Road, Ottawa, Ontario, Canada K1A 0R8

73. J. Alan George, Vice President, Academic and Provost, Needles Hall, University of Waterloo, Waterloo, Ontario, Canada N2L 3G1

74. John R. Gilbert, Xerox Palo Alto Research Center, 3333 Coyote Hill Road, Palo Alto, CA 94304

75. Gene H. Golub, Department of Computer Science, Stanford University, Stanford, CA 94305

76. Joseph F. Grcar, Division 8245, Sandia National Laboratories, Livermore, CA 94551-0969

77. John Gustafson, Ames Laboratory, Iowa State University, Ames, IA 50011

78. Michael T. Heath, National Center for Supercomputing Applications, 4157 Beckman Institute, University of Illinois, 405 North Mathews Avenue, Urbana, IL 61801-2300

79. Don E. Heller, Center for Research on Parallel Computation, Rice University, P.O. Box 1892, Houston, TX 77251

80. Dr. Dan Hitchcock, Office of Scientific Computing ER-7 Applied Mathematical Sciences, Office of Energy Research, U. S. Department of Energy, Washington DC 20585

81. Robert E. Huddleston, Computation Department, Lawrence Livermore National Laboratory, P.O. Box 808, Livermore, CA 94550

82. Dr. Gary Johnson, Office of Scientific Computing ER-7, Applied Mathematical Sciences, Office of Energy Research, U. S. Department of Energy, Washington DC 20585

83. Lennart Johnsson, Thinking Machines Inc., 245 First Street, Cambridge, MA 02142-1214

84. Harry Jordan, Department of Electrical and Computer Engineering, University of Colorado, Boulder, CO 80309

85. Malvyn H. Kalos, Cornell Theory Center, Engineering and Theory Center Bldg., Cornell University, Ithaca, NY 14853-3901

86. Hans Kaper, Mathematics and Computer Science Division, Argonne National Laboratory, 9700 South Cass Avenue, Bldg. 221, Argonne, IL 60439

87. Kenneth Kennedy, Department of Computer Science, Rice University, P.O. Box 1892, Houston, TX 77001

88. Thomas Kitchens, Department of Energy, Scientific Computing Staff, Office of Energy Research, ER-7, Office G-437 Germantown, Washington, DC 20585 
89. Richard Lau, Office of Naval Research, Code 1111MA, 800 Quincy Street, Boston, Tower 1, Arlington, VA 22217-5000

90. Alan J. Laub, Department of Electrical and Computer Engineering, University of California, Santa Barbara, CA 93106

91. Robert L. Launer, Army Research Office, P.O. Box 12211, Research Triangle Park, NC 27709

92. Charles Lawson, MS 301-490, Jet Propulsion Laboratory, 4800 Oak Grove Drive, Pasadena, CA 91109

93. Professor Peter Lax, Courant Institute for Mathematical Sciences, New York University, 251 Mercer Street, New York, NY 10012

94. John G. Lewis, Boeing Computer Services, P.O. Box 24346, M/S 7L-21, Seattle, WA 98124-0346

95. Robert F. Lucas, Supercomputer Research Center, 17100 Science Drive, Bowie, MD 20715-4300

96. Franklin Luk, Electrical Engineering Department, Cornell University, Ithaca, NY 14853

97. Paul C. Messina, Mail Code 158-79, California Institute of Technology, 1201 E. California Blvd., Pasadena, CA 91125

98. James McGraw, Lawrence Livermore National Laboratory, L-306, P.O. Box 808, Livermore, CA 94550

99. Cleve Moler, The Mathworks, 325 Linfield Place, Menlo Park, CA 94025

100. Dr. David Nelson, Director of Scientific Computing ER-7, Applied Mathematical Sciences, Office of Energy Research, U. S. Department of Energy, Washington DC 20585

101. Professor V. E. Oberacker, Department of Physics, Vanderbilt University, Box 1807 Station B, Nashville, TN 37235

102. Dianne P. O'Leary, Computer Science Department, University of Maryland, College Park, MD 20742

103. James M. Ortega, Department of Applied Mathematics, Thornton Hall, University of Virginia, Charlottesville, VA 22901

104. Charles F. Osgood, National Security Agency, Ft. George G. Meade, MD 20755

105. Roy P. Pargas, Department of Computer Science, Clemson University, Clemson, SC 29634-1906

106. Beresford N. Parlett, Department of Mathematics, University of California, Berkeley, CA 94720

107. Merrell Patrick, Department of Computer Science, Duke University, Durham, NC 27706

108. Robert J. Plemmons, Departments of Mathematics and Computer Science, Box 7311, Wake Forest University, Winston-Salem, NC 27109

109. James Pool, Caltech Concurrent Supercomputing Facility, California Institute of Technology, MS 158-79, Pasadena, CA 91125 
110. Alex Pothen, Department of Computer Science, Pennsylvania State University, University Park, PA 16802

111. Yuanchang Qi, IBM European Petroleum Application Center, P.O. Box 585, N4040 Hafrsfjord, Norway

112. Giuseppe Radicati, IBM European Center for Scientific and Engineering Computing, via del Giorgione 159, I-00147 Roma; Italy

113. Professor Daniel A. Reed, Computer Science Department, University of Illinois, Urbana, IL 61801

114. John K. Reid, Numerical Analysis Group, Central Computing Department, Atlas Centre, Rutherford Appleton Laboratory, Didcot, Oxon OX11 0QX, England

115. John R. Rice, Computer Science Department, Purdue University, West Lafayette, IN 47907

116. Donald J. Rose, Department of Computer Science, Duke University, Durham, NC 27706

117. Edward Rothberg, Department of Computer Science, Stanford University, Stanford, CA 94305

118. Joel Saltz, ICASE, MS 132C, NASA Langley Research Center, Hampton, VA 23665

119. Ahmed H. Sameh, Center for Supercomputer R\&D, 469 CSRL 1308 West Main St., University of Illinois, Urbana, IL 61801

120. Robert Schreiber, RIACS, Mail Stop 230-5, NASA Ames Research Center, Moffett Field, CA 94035

121. Martin H. Schultz, Department of Computer Science, Yale University, P.O. Box 2158 Yale Station, New Haven, CT 06520

122. David S. Scott, Intel Scientific Computers, 15201 N.W. Greenbrier Parkway, Beaverton, OR 97006

123. Kermit Sigmon, Department of Mathematics, University of Florida, Gainesville, FL 32611

124. Horst Simon, Mail Stop T045-1, NASA Ames Research Center, Moffett Field, CA 94035

125. Danny C. Sorensen, Department of Mathematical Sciences, Rice University, P. O. Box 1892, Houston, TX 77251

126. G. W. Stewart, Computer Science Department, University of Maryland, College Park, MD 20742

127. Paul N. Swartztrauber, National Center for Atmospheric Research, P.O. Box 3000, Boulder, CO 80307

128. Robert G. Voigt, ICASE, MS 132-C, NASA Langley Research Center, Hampton, VA 23665

129. Phuong Vu, Cray Research, Inc., 19607 Franz Rd., Houston, TX 77084

130. Robert Ward, Department of Computer Science, 107 Ayres Hall, University of Tennessee, Knoxville, TN 37996-1301 
131. Andrew B. White, Computing Division, Los Alamos National Laboratory, P.O. Box 1663 MS-265, Los Alamos, NM 87545

132. David Young, University of Texas, Center for Numerical Analysis, RLM 13.150, Austin, TX 78731

133. Office of Assistant Manager for Energy Research and Development, U.S. Department of Energy, Oak Ridge Operations Office, P.O. Box 2001 Oak Ridge, TN $37831-8600$

134-135. Office of Scientific \& Technical Information, P.O. Box 62, Oak Ridge, TN 37831 